\title{
植物功能性状与森林生态系统服务的关系研究综述
}

\author{
郑 华 ${ }^{1,2, *}$, 潘 权 ${ }^{1,2}$, 文 志 $^{1}$, 杨延征 ${ }^{1}$ \\ 1 中国科学院生态环境研究中心 城市与区域生态国家重点实验室,北京 100085 \\ 2 中国科学院大学 资源与环境学院, 北京 100049
}

\begin{abstract}
摘要:植物功能性状途径是揭示生物多样性与生态系统服务关系的重要视角,尽管植物功能性状与生态系统服务的关系在单一 地点的研究取得突出进展, 但对于植物功能性状与生态系统服务的关系仍缺乏整体认识。以森林生态系统为对象, 通过系统文 献检索及笁选, 收集了 216 篇文献, 应用整合分析和二分网络分析等方法, 探讨了植物功能性状对森林生态系统服务及其权衡 与协同关系的影响。结果表明:植物叶片功能性状关注最多,占研究性状数量的 $48 \%$,生态系统服务中关注最多是生物量、土壤 肥力、病虫害控制和固碳服务; $81.1 \%$ 的植物功能性状与生态系统服务关系组表现为稳定的正向或负向关系,而关联植物功能 性状多的生态系统服务(生物量、固碳服务、土壤水分、土壤肥力和病虫害控制)往往与植物功能性状表现为不稳定关系; 森林 生态系统中存在 6 组 “植物功能性状-生态系统服务簇(简称“性状-服务簇”) : 水循环相关的性状-服务簇、土壤保持相关的性 状-服务簇、物质生产相关的性状-服务簇、灾害控制相关的性状-服务簇、养分循环相关的性状-服务簇和授粉相关的性状-服务 簇, 揭示了各性状-服务簇内生态系统服务的权衡或协同关系以及与各性状-服务簇关系密切的植物功能性状。该研究从总体 上阐明了植物功能性状与森林生态系统服务关系的研究重点和进展、揭示了植物功能性状对森林生态系统服务影响效应的方 向和强度,可为深化森林生态系统服务形成机制认识以及协调生态系统服务权衡关系提供科学依据。
\end{abstract}

关键词: 植物功能性状;森林生态系统服务;生态系统功能;权衡;植物功能性状-生态系统服务簇

\section{Relationships between plant functional traits and ecosystem services in forests: A review}

Abstract: Plant functional traits provide an effective way to investigate the complex relationship between biodiversity and ecosystem services. Many empirical studies have revealed the importance of plant functional traits for ecosystem service delivery, but there is still a lack of general understanding of the relationship between plant functional traits and ecosystem services. Here a systematic analysis was performed to investigate the effects of plant functional traits on forest ecosystem services and their trade-off/synergy relationships based on 216 references by using meta-analysis and bipartite network analysis. Among plant functional traits, leaf functional traits are the most concerned, accounting for $48.0 \%$ of the studied plant functional traits. Biomass, soil fertility, biocontrol, and carbon sequestration are the most in-depth studies in ecosystem services. About $81.8 \%$ of plant functional traits have stable relationships (positive or negative) with forest ecosystem services, and the unstable relationship is mainly concentrated in the ecosystem services associated with multiple

基金项目: 国家自然科学基金项目 $(41925005$,41871217)

收稿日期:2021-03-27; 采用日期:2021-07-12

* 通讯作者 Corresponding author.E-mail: zhenghua@ rcees.ac.cn 
plant functional traits, such as biomass, carbon sequestration, soil water content, soil fertility, and herbivory and biocontrol. In forest ecosystem, six trait-service clusters are determined, namely, water-cycle-related cluster, soilconservation-related cluster, material-production-related cluster, disaster-control-related cluster, nutrient-cycle-related cluster, and pollination-related cluster, which reveal the relationships of trade-offs or synergies among forest ecosystem services. The key plant functional traits influencing these trait-service clusters were also identified. This study generally clarifies the research of the relationships between plant functional traits and forest ecosystem services, and reveals the influence direction and intensity of plant functional traits on forest ecosystems. The results can provide a scientific basis for understanding the formation mechanism of forest ecosystem services and coordinating the trade-off relationships of forest ecosystem services.

Key Words : plant functional trait; forest ecosystem service; ecosystem function; trade-off; trait-service cluster

生态系统服务是指自然生态系统在维持自身稳定的同时为人类提供的赖以生存的环境条件和效用 ${ }^{[1,2]}$, 它的形成与生态系统结构和过程密切相关, 越来越多的学者也趋于从生态系统服务形成过程去认识和分类生 态系统服务 (如: Common International Classification of Ecosystem Services, CICES ${ }^{[3]}$ ) $^{[4]}$, 但生态系统服务的形 成机制一直是生态系统服务研究的难点。近年来,随着植物功能性状研究的不断深人,直接影响植物生存、生 长和繁殖的形态、化学、生理以及物候特征 ${ }^{[5,6]}$ (即植物功能性状), 被认为是表征生态系统结构和功能的重要 指标。这些性状要么反映植物对环境变化的响应 (即响应性状), 要么决定植物对生态系统过程的影响 (即效 应性状 $)^{[5]}$ 。

植物功能性状能够驱动多种生态系统过程进而影响生态系统服务 ${ }^{[7,8]}$, 植物功能性状的优势度体现了物 种在群落中的资源获取能力, 而植物功能性状差异能够减少生态位的重叠, 并使其具有不同的资源获取策略, 从而提高资源利用的效率 ${ }^{[6]}$, 植物功能性状途径为揭示生态系统服务形成机制提供重要视角 ${ }^{[9]}$ 。尽管植物 功能性状与生态系统服务关系在单一地点的研究取得显著进展, 但对于植物功能性状与生态系统服务的关系 仍缺乏整体的认识。大量的研究表明植物功能性状是调控生态系统服务的重要因子 ${ }^{[8,10]}$, 但植物功能性状 与生态系统服务的关系具有不确定性, 不同的研究甚至可能会得出相反的结论, 例如: Aponte 等研究表明, 比 叶面积高的植物群落往往具有更高的生物量 ${ }^{[11]}$; 而 $\mathrm{Bu}$ 等研究表明, 比叶面积的增加不利于生态系统生物量 的积累 ${ }^{[12]}$; 还有研究结果显示比叶面积与生态系统生物量无关 ${ }^{[13]}$ 。

此外, 植物功能性状与生态系统服务之间存在着复杂的对应关系,一种生态系统服务受制于多种植物功 能性状的共同作用, 不同的生态系统服务往往会受共同的植物功能性状影响 ${ }^{[10,14]}$, 这些复杂的对应关系驱动 着生态系统服务之间的协同与权衡。为此, de Bello 等提出 “性状-服务簇” (trait-service clusters) 的概念, 将受 相同植物功能性状影响的生态系统服务定义为簇, 以理解植物功能性状与生态系统服务之间的复杂关系, 解 析生态系统服务协同与权衡机制, 并用于指导生态系统管理 ${ }^{[7]}$ 。Mario 等 ${ }^{[15]}$ 在其基础上, 通过对草地生态系 统中植物功能性状与生态系统服务的复杂关系进行网络分析, 并确定了 5 组 “性状-服务簇”, 并揭示驱动生态 系统服务权衡或协同关系的主要植物功能性状,为协调草地生态系统服务之间的权衡关系提供了管理依据。 “性状-服务簇” 的识别也有助于提高生态系统服务簇 ( ecosystem services bundles) 变化的预测能力 ${ }^{[9,16]}$ 。

森林生态系统作为全球陆地生态系统的重要组成部分, 在维持生物多样性和为人类提供生态系统服务方 面发挥着巨大作用。伴随着全球人口的不断增长和社会经济的高速发展, 人类对森林资源和森林生态系统服 务的需求快速增加, 导致森林生态系统面临的压力将越来越大, 森林生态系统健康和生态系统服务的供给能 力受到了严重影响, 严重威胁到人类社会的可持续发展 ${ }^{[17]}$, 揭示植物功能性状与森林生态系统服务关系, 具 有重要的理论与实践意义。本研究将以森林生态系统为研究对象, 使用整合分析的方法, 以探讨植物功能性 状与生态系统服务关系的数量特征。具体目标是: (1) 确定植物功能性状对森林生态系统服务影响效应的方 向和稳定性; (2) 探讨森林生态系统 “性状-服务簇” 中, 植物功能性状如何影响生态系统服务的协同与权衡? 


\section{1 数据来源与研究方法}

\section{1 文献检索}

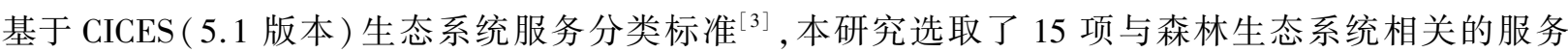
(表 1)。针对植物功能性状与森林生态系统服务,本研究分两步开展文献检索: 首先参考 van der Plas 的文献 检索的关键词 ${ }^{[18]}$, 设置了宽泛的关键词, 于 2020 年 5 月 24 日在 web of science 核心数据库中以主题检索的方 式进行了预检索。然后,基于预检索中出现的生态系统服务术语, 对这 15 项生态系统服务设置了精确的检索 关键词,于 2021 年 1 月 16 日再次进行检索,最终检索到 7120 篇文献。

表 115 种森林生态系统服务的类别

Table 1 Classification and connotation of 15 forest ecosystem services

\begin{tabular}{|c|c|c|c|}
\hline $\begin{array}{l}\text { 生态系统服务类别 } \\
\text { Ecosystem Service Types }\end{array}$ & $\begin{array}{l}\text { 生态系统服务 } \\
\text { Ecosystem services }\end{array}$ & $\begin{array}{l}\text { CICES 分类编号 } \\
\text { Code of CICES } \\
\text { division/group }\end{array}$ & $\begin{array}{l}\text { 关键词 } \\
\text { Keywords }\end{array}$ \\
\hline $\begin{array}{l}\text { 产品提供 } \\
\text { Provisioning }\end{array}$ & $\begin{array}{l}\text { 生物量 (BM) } \\
\text { Biomass }\end{array}$ & 1.1 & $\begin{array}{l}\text { "biomass" or "fuelwood" or " raw material" or " wood } \\
\text { production" or "timber production" }\end{array}$ \\
\hline \multirow[t]{13}{*}{$\begin{array}{l}\text { 调节与支持 } \\
\text { Regulating and supporting }\end{array}$} & $\begin{array}{l}\text { 土壤肥力 (SFE) } \\
\text { Soil fertility }\end{array}$ & 2.2.4.2 & $\begin{array}{l}\text { " soil fertility" or " soil nitrogen" or " soil phosphorus" } \\
\text { or " nitrogen retention" or "phosphorus retention" or " } \\
\text { nutrient retention" }\end{array}$ \\
\hline & $\begin{array}{l}\text { 径流控制 ( RC) } \\
\text { Runoff control }\end{array}$ & 2.2.1.3 & "flood" or "water regulation" or "runoff" \\
\hline & $\begin{array}{l}\text { 土壤水分 (SW) } \\
\text { Soil water content }\end{array}$ & $4.2 .2 .1-3$ & "soil water" or " soil moisture" or " Water retention" \\
\hline & $\begin{array}{l}\text { 水质净化 ( WTP) } \\
\text { Water purification }\end{array}$ & 2.1.1.1-2 & "nutrient reduce" or "waste * " or "toxic *" \\
\hline & $\begin{array}{l}\text { 病虫害控制( HC) } \\
\text { Herbivory and Pest control }\end{array}$ & 2.2.3.1 & $\begin{array}{l}\text { "herbivory" or "herbivores" or " natural enemy" or " } \\
\text { predators" or "parasites" or "pathogens" or " aphids" } \\
\text { or "leaf miner" }\end{array}$ \\
\hline & $\begin{array}{l}\text { 人侵控制 (IC) } \\
\text { Invasion control }\end{array}$ & 2.2.3.1 & "weed biomass" or "biomass variance" or "invasion" \\
\hline & $\begin{array}{l}\text { 固碳服务 }(\mathrm{CR}) \\
\text { Carbon regulation }\end{array}$ & 2.2.6.1 & $\begin{array}{l}\text { " carbon storage" or " carbon sequestration" or " soil } \\
\text { organic matter" or " soil organic carbon" or " litter } \\
\text { mass" or " litter stock" or " litter biomass" or " } \\
\text { litterfall" or "litter* depth" or " soil total carbon" }\end{array}$ \\
\hline & $\begin{array}{l}\text { 空气质量调节 }(A R) \\
\text { Regulation of temperature and humidity }\end{array}$ & 2.2.6.2 & $\begin{array}{l}\text { "air * temperature" or " air * humidity" or " cooling" } \\
\text { or "heat" or " microclimate" }\end{array}$ \\
\hline & $\begin{array}{l}\text { 土壤保持 }(\mathrm{EC}) \\
\text { Control of erosion rates }\end{array}$ & 2.2.1.1 & $\begin{array}{l}\text { "aggregate Stability" or " field holding capacity" or " } \\
\text { weight diameter" or " soil aggregate" or " Sediment } \\
\text { stability" or "soil detachment" }\end{array}$ \\
\hline & $\begin{array}{l}\text { 泥沙拦截 (BF) } \\
\text { Buffering and attenuation } \\
\text { of mass movement }\end{array}$ & 2.2.1.2 & $\begin{array}{l}\text { "sediment trap" or " hydraulic roughness" or " sediment } \\
\text { discharge" }\end{array}$ \\
\hline & $\begin{array}{l}\text { 授粉服务 (PN) } \\
\text { Pollination }\end{array}$ & 2.2 .2 .1 & $\begin{array}{l}\text { "pollinator" or " visitation rate" or " visitation } \\
\text { frequency" }\end{array}$ \\
\hline & $\begin{array}{l}\text { 火灾控制 }(\mathrm{FP}) \\
\text { Fire protection }\end{array}$ & 2.2.1.5 & $\begin{array}{l}\text { "burn time" or "fire sustainability" or " flame" or " } \\
\text { ignite" or "fire tolerance" or " heat release" or "burnt } \\
\text { biomass" }\end{array}$ \\
\hline & $\begin{array}{l}\text { 风害控制 (WP) } \\
\text { Wind protection }\end{array}$ & 2.2.1.4 & "wind damage" or " storm" or "hurricane" \\
\hline $\begin{array}{l}\text { 文化 } \\
\text { Cultural }\end{array}$ & $\begin{array}{l}\text { 文化服务 (CS) } \\
\text { Cultural service }\end{array}$ & 3.1.2.1-4 & $\begin{array}{l}\text { " recreation" or " Aesthetic" or " cultural" or " } \\
\text { perspective" }\end{array}$ \\
\hline
\end{tabular}

1.2 文献篮选和数据统计

首先, 通过阅读标题和摘要, 篮选出其中研究植物功能性状、植物功能多样性或生物多样性和生态系统功 
能或服务的文章; 然后, 通过阅读全文,并根据设置的 4 个标准,进一步精确篮选出报道植物功能性状与森林 生态系统服务关系文献,共篮选到符合要求的文献 216 篇。设置的 4 个篮选标准是: (1) 研究对象为森林生 态系统; (2) 研究的植物类型为陆地高等植物, 排除蒝类等低等植物的研究; (3) 实验为群落尺度的实地调查 研究,排除使用遥感数据或综述类的研究; (4) 研究内容为单个植物功能性状与生态系统服务的关系。

针对 216 篇文献中的植物功能性状与生态系统服务作用关系 (正向关系、负向关系、中性关系) 进行统 计, 收集到了群落水平的植物功能性状均值与生态系统服务的关系 1258 组, 其中, 在数据统计的过程中, 对于 多地点研究的文章, 则进行多次统计。尽管本研究设置了宽泛的预检索和精确检索, 但仍未发现在森林生态 系统中单个植物功能性状与生态系统文化服务关系的研究。最终统计了生物量、土壤肥力、径流控制、土壤水 分、水质净化、病虫害控制、人侵控制、固碳服务、空气质量调节、土壤保持、泥沙拦截、授粉服务、火灾控制以及 风害控制等 14 项服务, 共收集到 103 个植物功能性状, 在合并涵义类似(如比叶面积与比叶重) 或具有强相 关的性状 (如叶片光合速率与叶绿素含量) 后, 确定了本研究分析的 75 个植物功能性状。

\section{3 数据分析}

\subsection{1 植物功能性状对生态系统服务的影响效应}

在确定植物功能性状对森林生态系统服务的影响效应, 本研究首先剔除了只有一次研究的性状, 并且分 类变量 (如: 无性繁殖模型、根分布形态等) 也排除在外。然后按照 Harrison 等 ${ }^{[19]}$ 的方法计算了植物功能性状 对森林生态系统服务影响效应(Predominant direction)。

$$
P D=\frac{P A-N A}{T A}
$$

式中, $P A$ (Positive amount) 表示在该组植物功能性状与生态系统服务关系的研究中正向关系的数量, $N A$ (Negative amount) 表示负向关系的数量, $T A$ (Total amount) 表示所有关系(正向、负向以及中性关系)的数量。

1.3.2 植物功能性状-生态系统服务簇的网络分析

本研究采用二分网络分析方法 (bipartite network analysis) 确定植物功能性状与森林生态系统服务簇 ${ }^{[15]}$, 从而揭示植物功能性状对生态系统服务权衡与协同的影响。二分网络分析是用于探讨两组相互独立群体之 间的网络关系 ${ }^{[20]}$, 在生态学上, 常用于分析不同生物之间的相互作用关系 ${ }^{[21]}$ 。本研究基于植物功能性状与 森林生态系统服务之间的影响效应,在 R 语言中使用了“Bipartite” 包中的“metaComputeModules”方法进行网 络分析 ${ }^{[20,22]}$ 。

为减少数据的偏差, 本研究对原有数据进行了以下篮选标准: (1) 只考虑有效关系数量不低于该服务研 究总数量的 3\%的植物功能性状与服务的关系组; (2) 只考虑有确定影响效应的植物功能性状和服务的关系 组, 即影响效应的正值在 0.2 及以上的数据。最终保留了总共 128 组植物功能性状与生态系统服务的对应关 系。为保证结果的稳定性, 本研究进行了 30 次的重复运算, 然后选择似然性 (likelihood) 最高的结果。由于该 分析方法无法区分正负关系,因此在分析之前, 将影响效应一律转化为正值,但为体现 “植物功能性状与生态 系统服务簇” 内的权衡与协同关系, 本研究在结果中添加上了植物功能性状对生态系统服务影响的正负 效应。

\section{2 研究结果}

2.1 植物功能性状与森林生态系统服务关系研究的数量特征

在基于植物功能性状的生态系统服务研究中, 生物量的研究数量最多, 共发表了 72 篇文献, 占总文献数 量的 $1 / 3$; 其次是土壤肥力和病虫害控制服务,约占研究总文章数量的 $20 \%$; 另外有 35 篇文献报道了植物功 能性状与固碳服务之间的关系; 火灾控制、土壤水分以及土壤保持服务研究的数量不足整体的 $10 \%$,其中火 灾控制 16 篇、土壤水分 15 篇、土壤保持 11 篇; 而其他的调节服务如: 水质净化、空气质量调节、径流控制、风 害控制、泥沙拦截以及授粉服务的研究数量不足整体的 $5 \%$, 其中, 只有一篇研究报道了植物功能性状与水质 
净化服务的关系(图 1)。

216 篇文献共涉及 75 个植物功能性状 (其中有 17 个植物功能性状只报道一次, ) 和 4 个分类变量 (开花 时间、固氮能力、根分布形态、扩散繁殖方式) (图 2)。 研究最多的是叶片的性状, 占总体研究性状数量的 $48 \%$, 其形态性状和生理性状都有广泛研究, 研究最多 的形态性状是比叶面积、叶片面积以及叶片厚度;而研 究最多的生理性状是叶片的氮含量、干物质含量、磷含 量、碳氮含量比以及碳含量。在其他器官中, 研究最多 的是形态性状,例如: 在根系性状中, 比根长、根长密度、 根质量密度等形态性状研究多,而根系生理性状研究相 对较少; 在茎性状中, 研究最多的性状是木材密度; 在植 株性状中, 研究最多的是最大高度。另外有 16 篇文献 报道了种子质量与生态系统服务的关系。

\section{2 植物功能性状对森林生态系统服务的效应}

共统计了 206 组植物功能性状与生态系统服务的 影响关系。 $81.1 \%$ 的植物功能性状与生态系统服务关 系组表现为稳定的正向或负向关系,其中有 108 组关系 表现为稳定的正向关系 $(P D>=0.2)$, 有 59 组关系表现 为稳定的负向关系 $(\mathrm{PD}<=-0.2)$ 。此外, 39 组不稳定的 正负关系 $(-0.2<\mathrm{PD}<0.2)$ 主要存在于生物量、固碳服 务、土壤水分、土壤肥力、病虫害控制等关联植物功能性

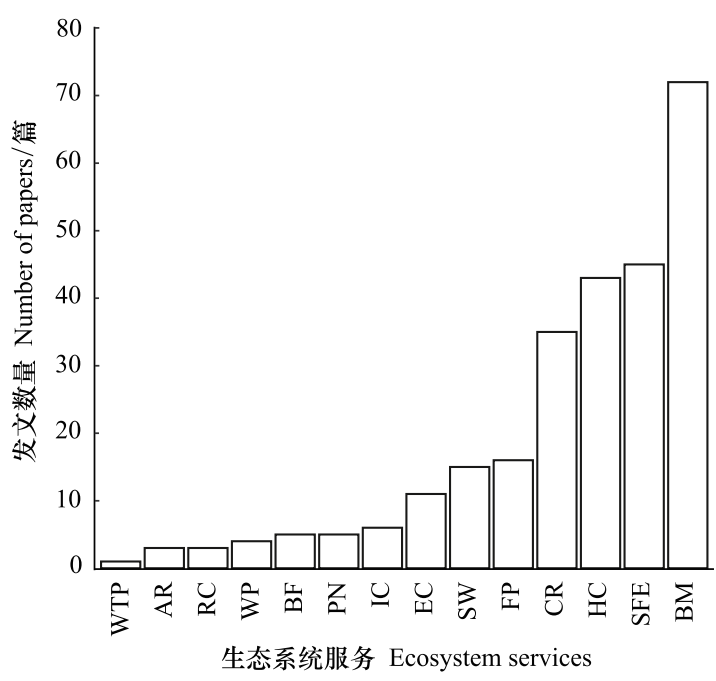

图 1 森林生态系统服务研究的发文数量

Fig.1 The spread of evidence on forest ecosystem service $\mathrm{AR}$ : 空气质量调节 Air quality regulationn; BF: 泥沙拦截 Buffering of sediment; BM:生物量 Biomass; $\mathrm{CR}$ : 固碳服务 Carbon regulation;EC: 土壤保持 Soil erosion control; FP: 火灾控制 Fire protection; HC: 病 虫害控制 Herbivory and pest control; IC: 人侵控制 Invasion control; $\mathrm{PN}$ : 授粉服务 Pollination; RC: 径流调节 Runoff control; SFE: 土壤肥 力 Soil fertility; SW: 土壤水分 Soil water content; WP: 风害控制 Wind protection;WTP : 水质净化 Water purification 状多的生态系统服务中 (图 3)。

单个植物功能性状往往与多种生态系统服务相关。 $84.9 \%$ 的植物功能性状关联多项生态系统服务, 仅有 $15.1 \%$ 的植物功能性状只影响一种生态系统服务。在与生态系统服务关系中, 研究较多的植物功能性状包括 叶的形态性状 (单叶面积、比叶面积) 和生理性状 (叶氮含量、叶碳含量、叶磷含量、叶干物质含量、叶光合速率 等)、细根百分比与根质量密度、木材密度、最大高度与冠层尺寸、种子质量 (图 3)。当单一植物功能性状影响 多项生态系统服务时, 植物功能性状是导致生态系统服务协同和权衡关系的主要因素, 例如: 具有高氮含量叶片 的植物群落具有更高的生物量以及更强的固碳和土壤肥力维持能力 ${ }^{[23]}$, 叶片氮含量成为促进这些生态系统协同 关系的主要因子; 而高木材密度能促进生物量的积累 ${ }^{[24]}$, 但也能降低了风害抵御能力 ${ }^{[25]}$, 木材密度就是导致生 物量累积和风害抵御服务权衡关系的因素。但有些植物功能性状只影响特定的生态系统服务,例如: 叶片的卷 曲度与火灾控制服务有关 ${ }^{[26]}$,树高茎比影响风害控制服务 ${ }^{[27]}$,根系的碳氮含量比与土壤肥力有关 ${ }^{[28]}$ 。

生态系统服务类型不同, 与之相关的植物功能性状也不相同。有的生态系统服务关联的植物功能性状 多,而有的生态系统服务关联的植物功能性状少。不同生态系统服务关联的植物功能性状有 2 到 34 个不等。 与土壤肥力相关的植物功能性状数量最多 (34 个), 影响生物量与固碳服务的植物功能性状数量也在 30 个以 上(图 3)。关联植物功能性状多的生态系统服务往往受植物多个器官的性状共同影响, 例如, 生物量不仅与 叶片的碳氮磷含量具有稳定的正向关系 ${ }^{[29]}$, 同时与茎的最大直径 ${ }^{[30]}$ 、木材密度 ${ }^{[31]}$ 、植株的最大高度以及种 子质量也与其存在正向关系 ${ }^{[32]}$ 。而关联植物功能性状数量较少的生态系统服务往往主要受特定器官的性状 的影响, 如空气调节服务主要与冠层大小有关 ${ }^{[33]}$, 土壤保持服务主要与根的形态性状有关, 而授粉服务仅与 花的大小和花的糖含量有关 ${ }^{[34,35]}$, 且主要为正向关系。

2.3 森林生态系统中的“植物功能性状-生态系统服务簇”

基于 47 个植物功能性状对 14 项生态系统服务影响效应的网络分析, 确定了 6 组植物功能性状与生态系 


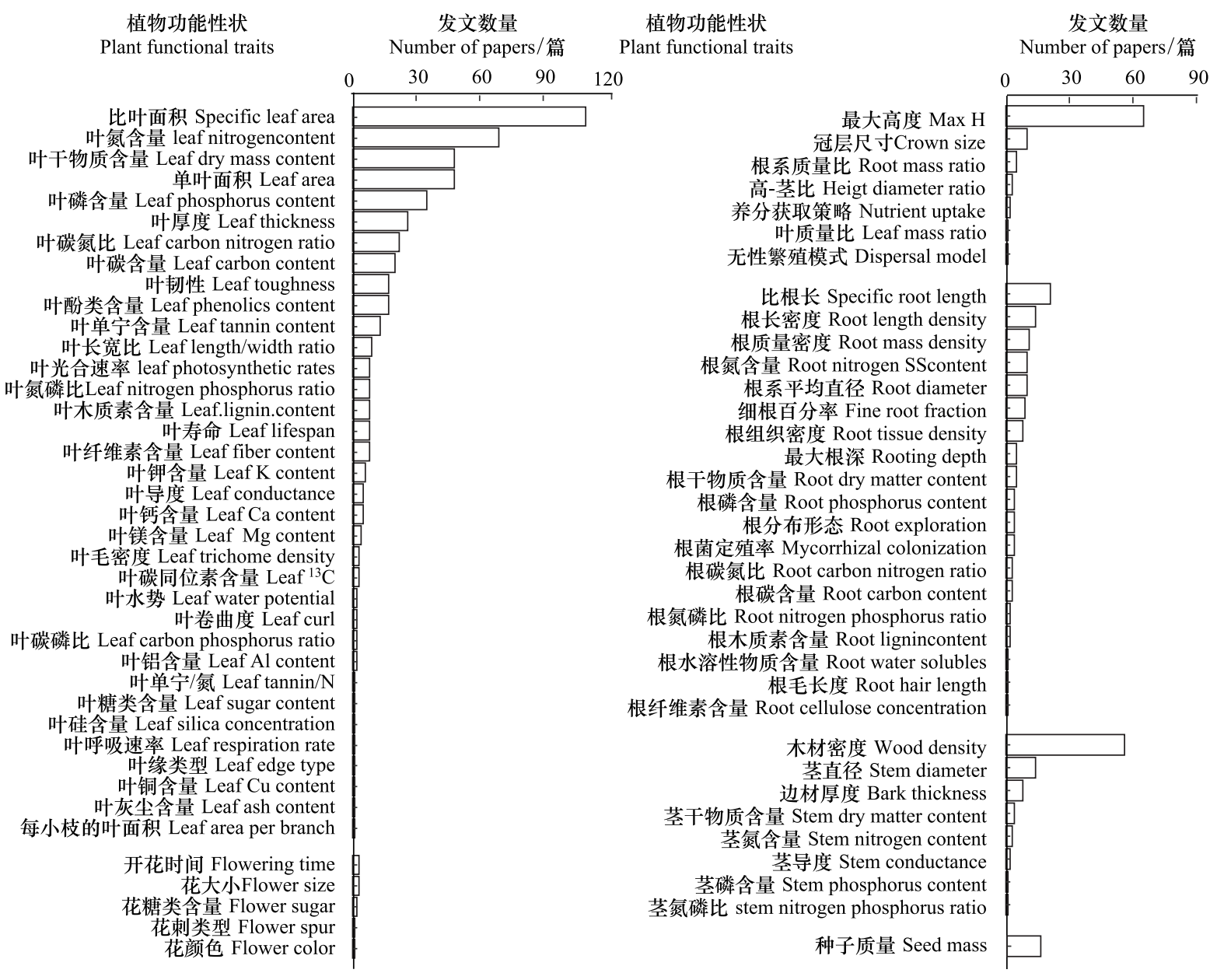

图 2 植物功能性状研究的发文数量

Fig.2 The spread of evidence on plant functional trait

统服务簇 (图 4)。其中, 水循环相关的性状服务簇、土壤保持相关的性状服务簇、物质生产相关的性状服务簇 以及灾害控制相关的性状服务簇中的植物功能性状能够驱动多种生态系统服务, 每个簇内的生态系统服务之 间呈现出权衡或协同的关系。

水循环相关的性状服务簇主要包括泥沙拦截、空气质量调节、土壤水分, 与其关系密切的植物功能性状有 单叶面积、茎导度、冠层尺寸、叶片导度和最大根深。在水循环相关服务簇中, 空气质量调节、土壤水分和泥沙 拦截之间主要存在着协同关系。

土壤保持相关的性状服务簇主要包括固碳服务、土壤保持和径流调节, 与其关系密切的植物功能性状有 叶片酚类含量、比根长、根氮含量、根质量密度、根干物质含量、根系平均直径、叶木质素含量、细根百分率、根 长密度、根木质素及含量以及根菌定殖率。在土壤保持相关服务簇中, 土壤保持、径流调节和固碳服务之间存 在着很强的协同关系。

物质生产相关的性状服务簇主要包括风害控制、人侵控制以及生物量, 与其关系密切的植物功能性状有 高径比、木材密度、茎氮含量、根系质量比、比叶面积、叶片氮含量、茎直径、最大高度、叶片碳氮比以及叶片光 合速率。在物质生产相关服务族中,生物量与人侵控制之间呈现出很强的协同关系, 与风害控制具有权衡关 系,但风害控制与人侵控制服务之间具有协同关系。 


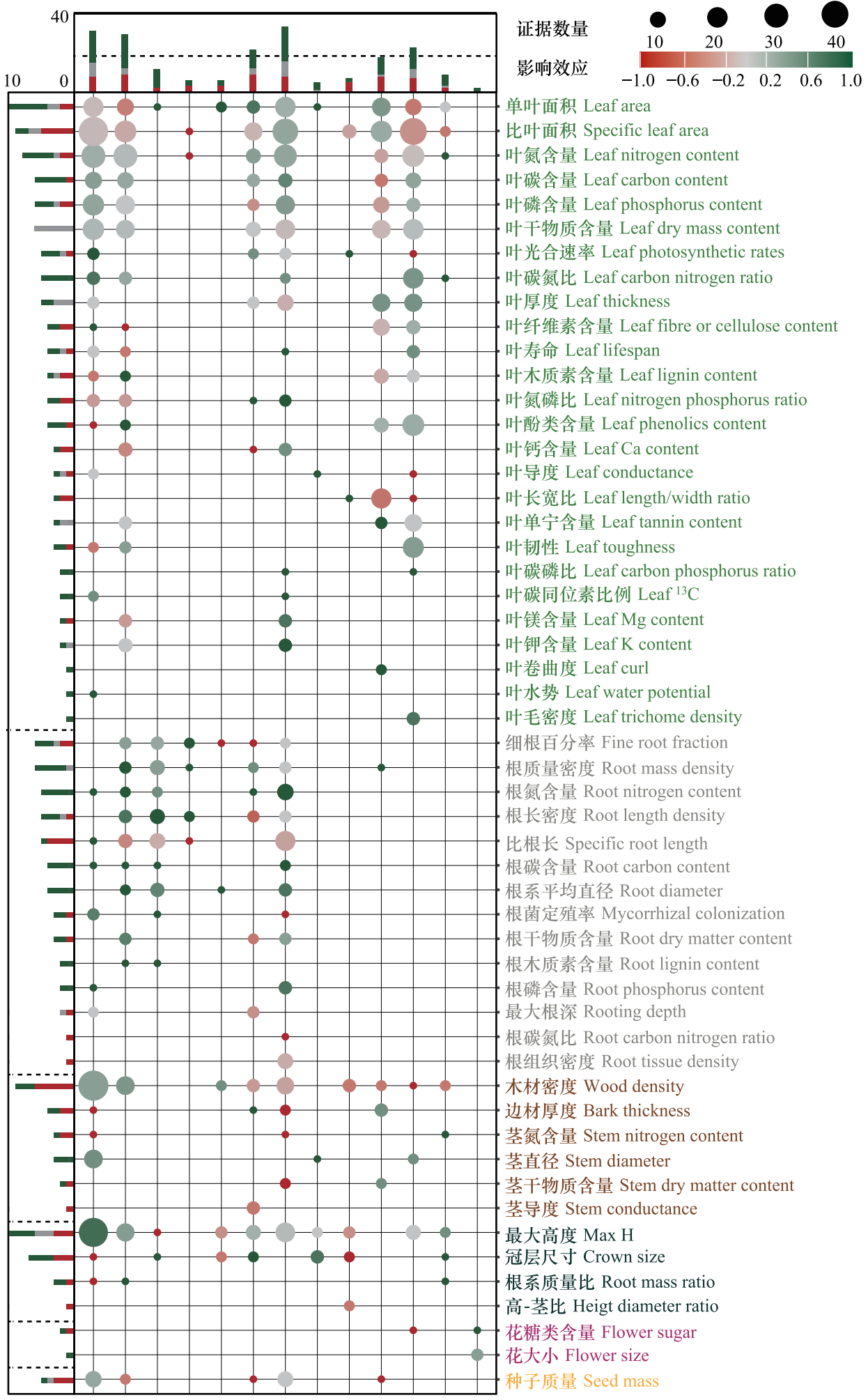

BM CR EC RC BF SW SFE AR WP FP HC IC PN

生态系统服务

Ecosystem services

植物功能性状

Plant functional traits

图 3 植物功能性状对生态系统服务的影响效应

Fig.3 Predominant direction of plant functional trait on forest ecosystem service

$\mathrm{AR}$ : 空气质量调节 Air quality regulationn; BF : 泥沙拦截 Buffering of sediment; BM:生物量 Biomass; CR: 固碳服务 Carbon regulation; EC: 土壤保 持 Soil erosion control;FP: 火灾控制 Fire protection; HC: 病虫害控制 Herbivory and pest control; IC: 入侵控制 Invasion control; PN: 授粉服务 Pollination;RC: 径流调节 Runoff control;SFE:土壤肥力 Soil fertility; SW : 土壤水分 Soil water content; WP: 风害控制 Wind protection 


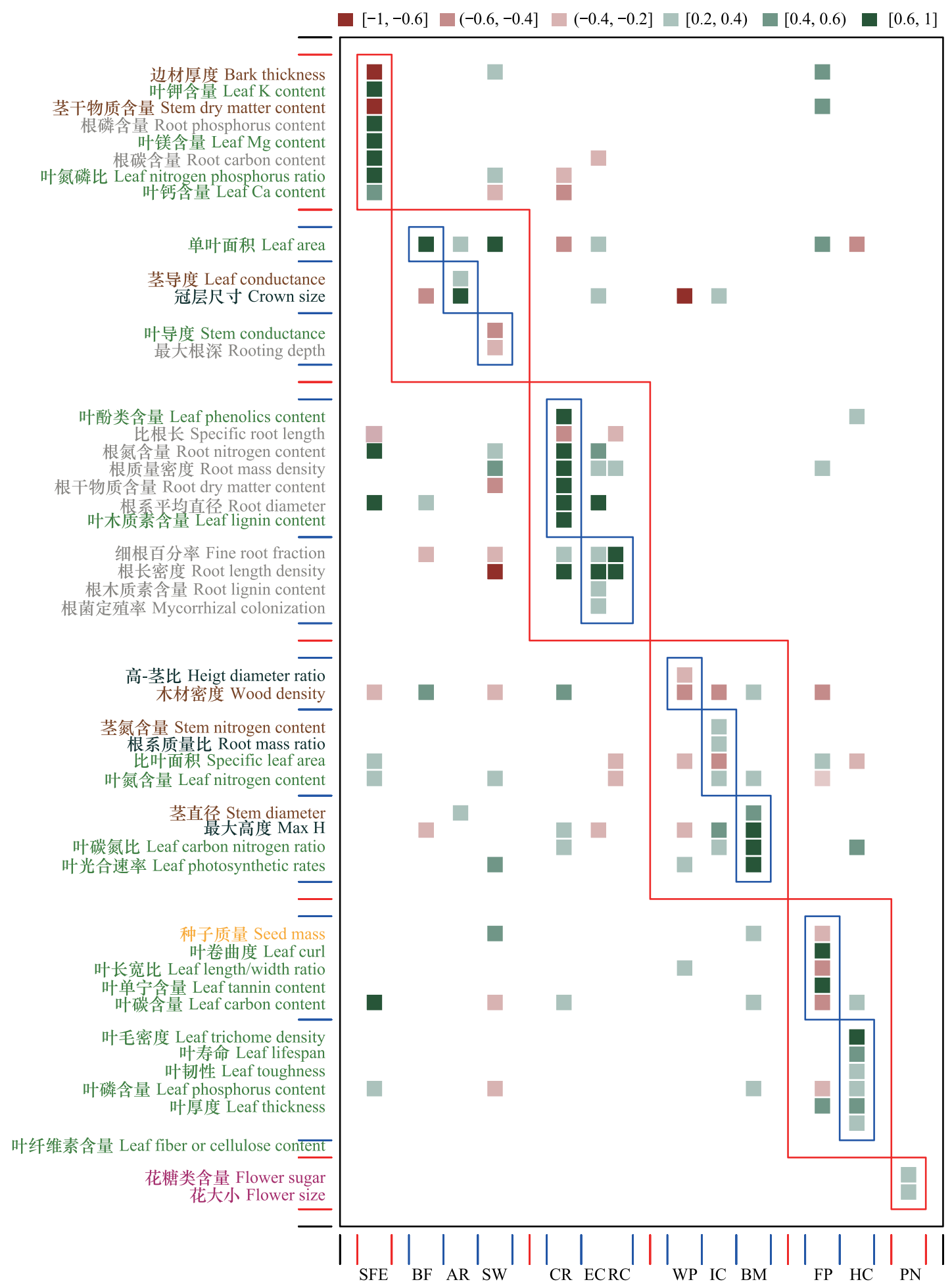

图 4 植物功能性状-生态系统服务簇的网络分析

Fig.4 The cluster of plant functional trait and forest ecosystem service

$\mathrm{AR}$ : 空气质量调节 Air quality regulationn; BF : 泥沙拦截 Buffering of sediment; BM:生物量 Biomass; CR: 固碳服务 Carbon regulation;EC:土壤保 持 Soil erosion control;FP: 火灾控制 Fire protection; HC: 病虫害控制 Herbivory and pest control; IC: 人侵控制 Invasion control; PN: 授粉服务 Pollination;RC: 径流调节 Runoff control;SFE:土壤肥力 Soil fertility; SW : 土壤水分 Soil water content;WP: 风害控制 Wind protection

灾害控制相关的性状服务簇主要包括火灾控制和病虫害控制, 与其关系密切的植物功能性状有种子质 量、叶片卷曲度、叶片长宽比、叶片单宁含量、叶片碳含量、叶毛密度、叶片寿命、叶片韧性、叶片磷含量、叶片厚 
度以及叶片纤维素含量。在灾害控制相关服务簇中,火灾控制和病虫害控制之间具有权衡关系。

养分循环相关的性状服务簇和授粉相关的性状服务簇的相关的性状组主要影响一种服务。与土壤肥力 关系密切的植物功能性状有边材厚度、叶片钾含量、茎干物质含量、根磷含量、叶片镁含量、根系碳含量、叶片 氮磷比以及叶片钙含量, 土壤肥力主要受元素含量相关性状的影响, 如根系碳含量、叶片氮磷比等 ${ }^{[36,37]}$ 。而 与授粉服务关系密切的植物功能性状主要为花的大小和花中糖类的含量。

\section{3 讨论}

通过系统地文献检索和整合分析, 本研究首次全面概述了植物功能性状与森林生态系统服务的相互关 系。结果表明,在本研究中检索到的 14 种森林生态系统服务的数量差异较大, 大多数研究主要关注容易观测 的服务,如生物量、土壤肥力、病虫害以及固碳服务等,而少有研究关注那些难以观测的服务,如: 水质净化、空 气质量调节以及径流控制等,这种服务研究数量的偏差在其他的整合分析中也同样存在 ${ }^{[19]}$ 。同样,在不同植 物器官之间, 叶片性状的研究远远多于其他性状的研究; 并且在同一植物器官内, 不同性状之间的研究数量也 存在差异, 软性状的研究数量也远多于硬性状 ${ }^{[7,15]}$ 。

3.1 植物功能性状与森林生态系统服务关系的不稳定性

基于已有文献统计的 206 组植物功能性状与生态系统服务的关系中, 有 59 组性状与服务之间具有不稳 定的正负关系,森林类型和环境因素可能是导致这些关系不稳定的重要因素。Fyllas 等 ${ }^{[38]}$ 在探究热带海拔梯 度上植物功能性状与森林净初级生产力关系时, 发现太阳辐射是影响植物功能性状与生态系统生产力之间的 关系重要因子; 同样, Buzzard 等 ${ }^{[39]}$ 研究表明,温度能够介导植物功能性状与生态系统土壤肥力之间的关系。 此外, 森林生态系统不同, 植物功能性状与生态系统服务的关系也可能发生变化, 例如, Abu 等 ${ }^{[40]}$ 在热带森林 的研究表明, 土壤肥力与叶片干物质含量之间具有正相关关系, 而 Laughlin 等 ${ }^{[41]}$ 在温带森林的研究得出了相 反的结论。

3.2 植物功能性状对生态系统服务权衡协同关系的影响

单个植物功能性状往往关联多种生态系统服务, 并决定了生态系统服务之间的权衡或协同关系 ${ }^{[15]}$ 。基 于植物功能性状与生态系统服务的影响效应的网络分析结果表明,森林生态系统中有 4 组具有权衡协同关系 的“植物功能性状-生态系统服务簇”。在水循环相关的生态系统服务簇中, 叶片的面积是影响泥沙拦截服务 的主要植物功能性状 ${ }^{[42]}$, 但同时对空气质量调节和土壤水分也具有正向的作用 ${ }^{[33,43]}$; 而冠层尺寸更大的植 物群落能够增加生态系统空气质量调节服务 ${ }^{[44]}$, 但降低了泥沙拦截服务 ${ }^{[45]}$ 。在土壤保持相关的生态系统服 务簇中, 高的根系质量密度、细根百分率和根长密度对这三种服务都具有促进作用 ${ }^{[46]}$, 另外高的根系氮含量 和根系直径也能提高固碳能力和土壤保持服务 ${ }^{[46,47]}$, 而比根长增加会降低固碳服务和土壤保持服务 ${ }^{[46,48]}$ 。 在物质生产相关的生态系统服务簇中, 低的木材密度和比叶面积都有利于提高生态系统风害控制和人侵控制 服务 ${ }^{[34,43]}$, 而高的叶片氮含量、最大高度和叶片碳氮比对生态系统生物量和人侵控制都具有积极作用 ${ }^{[43-46]}$ 。 在灾害控制相关的生态系统服务簇中, 高叶片碳含量和磷含量能够增加生态系统的病虫害控制能力 ${ }^{[47,48]}$, 而 降低了火灾抵御的能力 ${ }^{[49]}$; 但叶片厚度的增加能够同时提高生态系统的火灾控制和病虫害控制服务 ${ }^{[50,51]}$ 。

3.3 植物功能性状与生态系统服务关系对生态系统管理的启示

在生态系统管理中, 合理配置群落的物种组成是协调生态系统服务的权衡关系的重要途径 ${ }^{[49]}$, “植物功 能性状-生态系统服务簇”为物种配置提供了重要依据。在协调同一簇内的生态系统服务之间的权衡关系时， 应该优先考虑影响该簇内生态系统服务的主要植物功能性状, 如在水循环相关服务簇中, 应该优先将叶片的 面积和冠层尺寸作为物种选择的主要依据; 而对于不同服务簇之间的服务应该优先考虑服务簇之外的性状， 如在生物量和土壤水分之间,应该优先将冠层尺寸、木材密度和叶片光合速率作为物种选择的主要依据。另 外, 植物功能性状的多样性也是影响生态系统服务的重要维度, 有研究表明: 比叶面积的大小差异对群落生物 量积累具有正向作用 ${ }^{[50]}$, 叶片干物质含量的差异对群落生物量具有负效应 ${ }^{[51]}$ 。由于植物功能性状差异对生 
态系统服务影响的研究较少, 本研究只能限于群落水平的植物功能性状均值对生态系统服务的影响。但在物 种选择和丰富度配置过程中,植物功能性状的差异也应该被考虑。此外, “植物功能性状-生态系统服务簇” 在 景观尺度上也具有一定的适用性,例如,在土壤保持相关的生态系统服务簇中, 土壤保持、固碳和径流控制服 务表现出很强的协同关系,这与祁宁等人在中国东北地区基于服务簇的研究结果一致 ${ }^{[52]}$ 。

在今后的研究中, 植物功能性状与森林生态系统服务之间的复杂的相互作用关系的研究在以下方面值得 进一步深化: 加大植物功能性状对水质净化、空气质量调节、径流控制、风害控制、泥沙拦截以及授粉服务影响 的研究; 在植物功能性状选择方面, 应更加关注除叶片外其他器官的性状, 并且将植物功能性状的差异纳人研 究中; 植物功能性状之间的相互关系如何影响生态系统服务; 如何将植物功能性状纳人生态系统服务量化评 估中, 并建立基于植物功能性状与生态系统服务关系的生态系统评估方法; 如何将植物功能性状与生态系统 服务的关系应用到实际中, 并将生态系统尺度和景观尺度的生态系统管理措施相结合已解决生态修复和管理 等方面的系统问题。

\section{参考文献 (References) :}

[ 1 ] Daily G C. Nature's Services: Societal Dependence on Natural Ecosystems. Washington: Island Press, 1997.

[ 2 ] MEA. Millennium Ecosystem Assessment. Ecosystems and Human Well-Being: Synthesis Report. Washington: Island Press, 2005.

[ 3 ] Haines-Young R, Potschin M. Common International Classification of Ecosystem Services (CICES) V5.1. Guidance on the Application of the Revised Structure. Barton: Fabis Consult, 2018.

[ 4 ] Finisdore J, Rhodes C, Haines-Young R, Maynard S, Wielgus J, Dvarskas A, Houdet J, Quetier F, Lamothe K A, Ding H, Soulard F, Van Houtven G, Rowcroft P. The 18 benefits of using ecosystem services classification systems. Ecosystem Services, 2020, 45: 101160.

[ 5 ] Violle C, Navas M L, Vile D, Kazakou E, Fortunel C, Hummel I, Garnier E. Let the concept of trait be functional! Oikos, 2007, 116( 5): 882-892.

[ 6 ] 刘晓娟, 马克平. 植物功能性状研究进展. 中国科学: 生命科学, 2015, 45 (4): 325-339.

[ 7 ] De Bello F, Lavorel S, Diaz S, Harrington R, Cornelissen J H C, Bardgett R D, Berg M P, Cipriotti P, Feld C K, Hering D, Da Silva P M, Potts S G, Sandin L, Sousa J P, Storkey J, Wardle D A, Harrison P A. Towards an assessment of multiple ecosystem processes and services via functional traits. Biodiversity and Conservation, 2010, 19(10): 2873-2893.

[ 8 ] Liu C C, Li Y, Yan P, He N P. How to improve the predictions of plant functional traits on ecosystem functioning? Frontiers in Plant Science, 2021, 12: 3389 .

[ 9 ] Lamarque P, Lavorel S, Mouchet M, Quétier F. Plant trait-based models identify direct and indirect effects of climate change on bundles of grassland ecosystem services. Proceedings of the National Academy of Sciences of the United States of America, 2014, 111(38) : 13751-13756.

[10］潘权, 郑华, 王志恒, 文志, 杨延征. 植物功能性状对生态系统服务影响研究进展. 植物生态学报, 2020 (2021-04-01). https://kns.cnki. net $/$ KCMS/detail/detail. aspx? dbcode $=$ CJFQ\&dbname $=$ CAPJLAST\&filename $=$ ZWSB20210401001\&V $=$ MDg3OTdqNTdUM2ZscVdNMENMTDdSN3FkWnVacUZDemxWYjdPSIZvPVB6clliTEc0SE5ETXE0OUVaT3NPWXc5TXptUm42.

[11] Aponte C, Kasel S, Nitschke C R, Tanase M A, Vickers H, Parker L, Fedrigo M, Kohout M, Ruiz-Benito P, Zavala M A, Bennett L T. Structural diversity underpins carbon storage in Australian temperate forests. Global Ecology and Biogeography, 2020, 29(5) : 789-802.

[12] Bu W S, Huang J H, Xu H, Zang R G, Ding Y, Li Y D, Lin M X, Wang J S, Zhang C C. Plant functional traits are the mediators in regulating effects of abiotic site conditions on aboveground carbon stock-evidence from a 30 ha tropical forest plot. Frontiers in Plant Science, 2019, 9: 1958.

[13] Prado J A, Schiavini I, Vale V S, Arantes C S, Van Der Sande M T, Lohbeck M, Poorter L. Conservative species drive biomass productivity in tropical dry forests. Journal of Ecology, 2016, 104(3): 817-827.

[14] Cardinale B J, Duffy J E, Gonzalez A, Hooper D U, Perrings C, Venail P, Narwani A, Mace G M, Tilman D, Wardle D A, Kinzig A P, Daily G C, Loreau M, Grace J B, Larigauderie A, Srivastava D S, Naeem S. Biodiversity loss and its impact on humanity. Nature, 2012, 486( 7401) : 5967.

[15] Hanisch M, Schweiger O, Cord A F, Volk M, Knapp S. Plant functional traits shape multiple ecosystem services, their trade-offs and synergies in grasslands. Journal of Applied Ecology, 2020, 57(8) : 1535-1550.

[16] Raudsepp-Hearne C, Peterson G D, Bennett E M. Ecosystem service bundles for analyzing tradeoffs in diverse landscapes. Proceedings of the National Academy of Sciences of the United States of America, 2010, 107(11): 5242-5247.

[17] 刘世荣, 代力民, 温远光, 王晖. 面向生态系统服务的森林生态系统经营: 现状、挑战与展望. 生态学报, 2015, 35(1) : 1-9. 
[18] Van Der Plas F. Biodiversity and ecosystem functioning in naturally assembled communities. Biological Reviews, 2019, 94(4): 1220-1245.

[19] Harrison P A, Berry P M, Simpson G, Haslett J R, Blicharska M, Bucur M, Dunford R, Egoh B, Garcia-Llorente M, Geamana N, Geertsema W, Lommelen E, Meiresonne L, Turkelboom F. Linkages between biodiversity attributes and ecosystem services : a systematic review. Ecosystem Services, 2014, 9: 191-203.

[20] Carsten F. Dormann J F, Blüthgen N, Gruber B. Indices, graphs and null models: analyzing bipartite ecological networks. The Open Ecology Journal, 2009, 2(1): 7-24.

[21] Feng K, Zhang Y G, He Z L, Ning D L, Deng Y. Interdomain ecological networks between plants and microbes. Molecular Ecology Resources, $2019,19(6): 1565-1577$.

[22] R Core Team. R: A Language and Environment for Statistical Computing. Vienna, Austria: R Foundation for Statistical Computing. Vienna, Austria, 2019.

[23] Osborne B B, Nasto M K, Soper F M, Asner G P, Balzotti C S, Cleveland C C, Taylor P G, Townsend A R, Porder S. Leaf litter inputs reinforce islands of nitrogen fertility in a lowland tropical forest. Biogeochemistry, 2020, 147(3) : 293-306.

[24] Yuan Z Q, Ali A, Wang S P, Gazol A, Freckleton R, Wang X G, Lin F, Ye J, Zhou L, Hao Z Q, Loreau M. Abiotic and biotic determinants of coarse woody productivity in temperate mixed forests. Science of the Total Environment, 2018, 630: 422-431.

[25] Jimenez-Rodriguez D L, Alvarez-Anorve M Y, Pineda-Cortes M, Flores-Puerto J I, Benitez-Malvido J, Oyama K, Avila-Cabadilla L D. Structural and functional traits predict short term response of tropical dry forests to a high intensity hurricane. Forest Ecology and Management, 2018, 426: $101-114$.

[26] Burton J E, Cawson J G, Filkov A I, Penman T D. Leaf traits predict global patterns in the structure and flammability of forest litter beds. Journal of Ecology, 2021, 109(3): 1344-1355.

[27] Paz H, Vega-Ramos F, Arreola-Villa F. Understanding hurricane resistance and resilience in tropical dry forest trees: a functional traits approach. Forest Ecology and Management, 2018, 426: 115-122.

[28] Hertel D. Tree roots in canopy soils of old European beech trees-an ecological reassessment of a forgotten phenomenon. Pedobiologia, 2011, 54(2) : $119-125$.

[29] Rawat M, Arunachalam K, Arunachalam A, Alatalo J M, Kumar U, Simon B, Hufnagel L, Micheli E, Pandey R. Relative contribution of plant traits and soil properties to the functioning of a temperate forest ecosystem in the Indian Himalayas. Catena, $2020,194: 104671$.

[30 ] Jucker T, Sanchez A C, Lindsell J A, Allen H D, Amable G S, Coomes D A. Drivers of aboveground wood production in a lowland tropical forest of West Africa: teasing apart the roles of tree density, tree diversity, soil phosphorus, and historical logging. Ecology and Evolution, 2016, 6( 12) : 4004-4017.

[31] Mensah S, Salako K V, Assogbadjo A, Kakai R G, Sinsin B, Seifert T. Functional trait diversity is a stronger predictor of multifunctionality than dominance: evidence from an afromontane forest in South Africa. Ecological Indicators, 2020, 115: 106415.

[32] Falster D S, Brannstrom A, Dieckmann U, Westoby M. Influence of four major plant traits on average height, leaf-area cover, net primary productivity, and biomass density in single-species forests: a theoretical investigation. Journal of Ecology, 2011, 99(1) : 148-164.

[33] Speak A, Montagnani L, Wellstein C, Zerbe S. The influence of tree traits on urban ground surface shade cooling. Landscape and Urban Planning, 2020, 197: 103748 .

[34] Temeles E J, Bishop G A. A hurricane alters pollinator relationships and natural selection on an introduced island plant. Biotropica, 2019, 51(2) : $129-138$.

[35] Maruyama P K, Oliveira G M, Ferreira C, Dalsgaard B, Oliveira P E. Pollination syndromes ignored: importance of non-ornithophilous flowers to neotropical savanna hummingbirds. Naturwissenschaften, 2013, 100(11): 1061-1068.

[36] Liu J G, Gou X H, Gunina A, Long X E, Zhang F, Zhang J Z. Soil nitrogen pool drives plant tissue traits in alpine treeline ecotones. Forest Ecology and Management, 2020, 477: 118490.

[37] Ding J X, Kong D L, Zhang Z L, Cai Q, Xiao J, Liu Q, Yin H J. Climate and soil nutrients differentially drive multidimensional fine root traits in ectomycorrhizal-dominated alpine coniferous forests. Journal of Ecology, 2020, 108(6) : 2544- 2556.

[38 Fyllas N M, Bentley L P, Shenkin A, Asner G P, Atkin O K, Diaz S, Enquist B J, Farfan-Rios W, Gloor E, Guerrieri R, Huasco W H, Ishida Y, Martin R E, Meir P, Phillips O, Salinas N, Silman M, Weerasinghe L K, Zaragoza-Castells J, Malhi Y. Solar radiation and functional traits explain the decline of forest primary productivity along a tropical elevation gradient. Ecology Letters, 2017, 20(6) : 730-740.

[39] Buzzard V, Michaletz S T, Deng Y, He Z L, Ning D L, Shen L N, Tu Q C, Van Nostrand J D, Voordeckers J W, Wang J J, Weiser M D, Kaspari M, Waide R B, Zhou J Z, Enquist B J. Continental scale structuring of forest and soil diversity via functional traits. Nature Ecology \& Evolution, 2019, 3(9): 1298-1308.

[40] Hanif M A, Yu Q S, Rao X Q Shen W J. Disentangling the contributions of plant taxonomic and functional diversities in shaping aboveground 
biomass of a restored forest landscape in Southern China. Plants, 2019, 8(12): 612 .

[41] Laughlin D C, Richardson S J, Wright E F, Bellingham P J. Environmental filtering and positive plant litter feedback simultaneously explain correlations between leaf traits and soil fertility. Ecosystems, 2015, 18(7): 1269-1280.

[42] Coelho M S, Carlos P P, Pinto V D, Meireles A, Negreiros D, Morellato L P C, Fernandes G W. Connection between tree functional traits and environmental parameters in an archipelago of montane forests surrounded by rupestrian grasslands. Flora, 2018, 238 : 51-59.

[43] Vasquez-Valderrama M, Gonzalez-M R, Lopez-Camacho R, Baptiste M P, Salgado-Negret B. Impact of invasive species on soil hydraulic properties: importance of functional traits. Biological Invasions, 2020, 22(6) : 1849-1863.

[44] Helletsgruber C, Gillner S, Gulyás Á, Junker R R, Tanács E, Hof A. Identifying tree traits for cooling urban heat islands-a cross-city empirical analysis. Forests, 2020, 11(10): 1064 .

[45] Seitz S, Goebes P, Song Z, Bruelheide H, Härdtle W, Kühn P, Li Y, Schohen T. Tree species and functional traits but not species richness affect interrill erosion processes in young subtropical forests. Soil, 2016, 2(1): 49-61.

[46] Hao H X, Di H Y, Jiao X, Wang J G, Guo Z L, Shi Z H. Fine roots benefit soil physical properties key to mitigate soil detachment capacity following the restoration of eroded land. Plant and Soil, 2020, 446(1-2) : 487-501.

[47] Chen Z J, Zhou X Y, Geng S C, Miao Y, Cao Y H, Chen Z, Zhang J H, Han S J. Interactive effect of nitrogen addition and throughfall reduction decreases soil aggregate stability through reducing biological binding agents. Forest Ecology and Management, 2019 , 445: $13-19$.

[48] Demenois J, Rey F, Ibanez T, Stokes A, Carriconde F. Linkages between root traits, soil fungi and aggregate stability in tropical plant communities along a successional vegetation gradient. Plant and Soil, 2018, 424(1-2) : 319-334.

[49] Zheng H, Wang L J, Wu T. Coordinating ecosystem service trade-offs to achieve win-win outcomes: a review of the approaches. Journal of Environmental Sciences, 2019, 82: 103-112.

[50] Tahmasebi P, Moradi M, Omidipour R. Plant functional identity as the predictor of carbon storage in semi-arid ecosystems. Plant Ecology \& Diversity, 2017, 10(2-3): 139-151.

[51] Yi S J, Wu P, Peng X Q, Bai F H, Gao Y N, Zhang W X, Du N, Guo W H. Functional identity enhances aboveground productivity of a coastal saline meadow mediated by Tamarix chinensis in Laizhou Bay, China. Scientific Reports, 2020, 10(1): 5826.

[52] 祁宁, 赵君, 杨延征, 苟睿坤, 陈佳琦, 赵鹏祥, 李卫忠. 基于服务簇的东北地区生态系统服务权衡与协同. 生态学报, 2020, 40(9)： 2827-2837. 